OPEN ACCESS

Edited by:

Chao Wang,

Soochow University, China

Reviewed by:

Cong Luo,

Shenyang Pharmaceutical University,

China

Mingzhen Zhang,

Xi'an Jiaotong University, China

${ }^{*}$ Correspondence:

Jing Huang

huangjing0722hh@126.com

Honglin Jin

jin@hust.edu.cn

${ }^{+}$These authors have contributed equally to this work and share first authorship

Specialty section:

This article was submitted to

Cancer Immunity and Immunotherapy,

a section of the journal

Frontiers in Immunology

Received: 10 December 2021 Accepted: 27 December 2021

Published: 17 January 2022

Citation:

Liu Y, Geng Y, Yue B, Lo P-C, Huang $J$ and Jin $H$ (2022) Injectable Hydrogel as a Unique Platform for Antitumor Therapy

Targeting Immunosuppressive Tumor Microenvironment.

Front. Immunol. 12:832942. doi: 10.3389/fimmu.2021.832942

\section{Injectable Hydrogel as a Unique Platform for Antitumor Therapy Targeting Immunosuppressive Tumor Microenvironment}

\author{
Yushuai Liu ${ }^{1 \dagger}$, Yuanyuan Geng ${ }^{2 \dagger}$, Beilei Yue ${ }^{2}$, Pui-Chi Lo ${ }^{3}$, Jing Huang ${ }^{2 *}$ \\ and Honglin $\mathrm{Jin}^{2 *}$
}

1 Department of Ophthalmology, Union Hospital, Tongji Medical College, Huazhong University of Science and Technology, Wuhan, China, ${ }^{2}$ College of Biomedicine and Health and College of Life Science and Technology, Huazhong Agricultural University, Wuhan, China, ${ }^{3}$ Department of Biomedical Sciences, City University of Hong Kong, Kowloon, Hong Kong, SAR China

Cancer immunotherapy can boost the immune response of patients to eliminate tumor cells and suppress tumor metastasis and recurrence. However, immunotherapy resistance and the occurrence of severe immune-related adverse effects are clinical challenges that remain to be addressed. The tumor microenvironment plays a crucial role in the therapeutic efficacy of cancer immunotherapy. Injectable hydrogels have emerged as powerful drug delivery platforms offering good biocompatibility and biodegradability, minimal invasion, convenient synthesis, versatility, high drug-loading capacity, controlled drug release, and low toxicity. In this review, we summarize the application of injectable hydrogels as a unique platform for targeting the immunosuppressive tumor microenvironment.

Keywords: cancer immunotherapy, tumor microenvironment (TME), injectable hydrogels, immunogenic cell death, abscopal effect, controlled drug release

\section{INTRODUCTION}

Cancer is a major threat to human health worldwide (1). Cancer immunotherapy has emerged as a promising cancer treatment approach that can inhibit tumor metastasis and recurrence by boosting antitumor immune responses $(2,3)$. Cancer immunotherapies have revolutionized the treatment of many cancer types in clinical settings. Immunotherapeutic agents include immune checkpoint inhibitors, vaccines, immunologic adjuvants, adoptive cell transfer, and nonspecific immunestimulating factors (e.g., cytokines) (4). Nevertheless, low T cell infiltration levels, the presence of inhibitory immune cells, and the lack of neoantigens limit response to immunotherapy. Systemic administration of conventional drugs often requires high dosages or multiple injections, which can lead to severe immune-related adverse effects and low patient compliance (5-7). Multiple immunosuppressive factors in the tumor microenvironment (TME) have been shown to affect the delivery of therapeutic agents and efficacy of $T$ cell-based therapies, thus influencing the therapeutic efficacy of cancer immunotherapy (8-10). Therefore, modulating or reprogramming the 
immunosuppressive TME can enhance the efficacy of cancer immunotherapy. Many studies and clinical trials aiming to target tumor immunosuppressive microenvironment to eradicate malignant cells are ongoing $(10,11)$.

Hydrogels with 3D network structures have been widely used in various fields, especially in biomedicine $(7,12-14)$. Injectable hydrogels have attracted considerable attention as vehicles for sustained drug delivery in situ because of their unique advantages, including easy delivery by syringe and minimal surgical wounds $(13,15)$. Injectable hydrogels can be loaded with various agents, including chemotherapeutic drugs, immunotherapeutic agents, antibodies, vaccines, cytokines, and immune cells $(7,14,16)$. Sustained and controlled release of these therapeutic agents by injectable hydrogels can activate systemic antitumor immune responses and inhibit tumor metastasis and recurrence while causing minimal toxicity (7). Herein, we highlight recent advances in reprogramming the immunosuppressive TME using injectable hydrogels to improve the efficacy of cancer immunotherapy (Figure 1).

\section{CATEGORIES OF INJECTABLE HYDROGELS}

Injectable hydrogels are usually formed by quick sol-gel phase transition or chemical polymerization in situ. They can be directly delivered into the target sites by injection $(12,16)$. Injectable hydrogels can be classified into chemically and physically cross-linked hydrogels based on the gelling mechanism $(13,16)$. Chemically cross-linked injectable hydrogels are generated by introducing covalent linkages between polymer chains via disulfide formation, photoirradiation, enzymes, Schiff's base reactions, Michael-type addition reactions, or Diels-Alder reactions (16). On the other hand, physically cross-linked injectable hydrogels are formed through intermolecular interactions, such as hydrogen bonds, hydrophobic interactions, ionic cross-linking, and host-guest interaction (16). Injectable hydrogels can also be classified as natural or synthetic hydrogels based on the polymers used for their preparation (7). Natural injectable hydrogels are typically

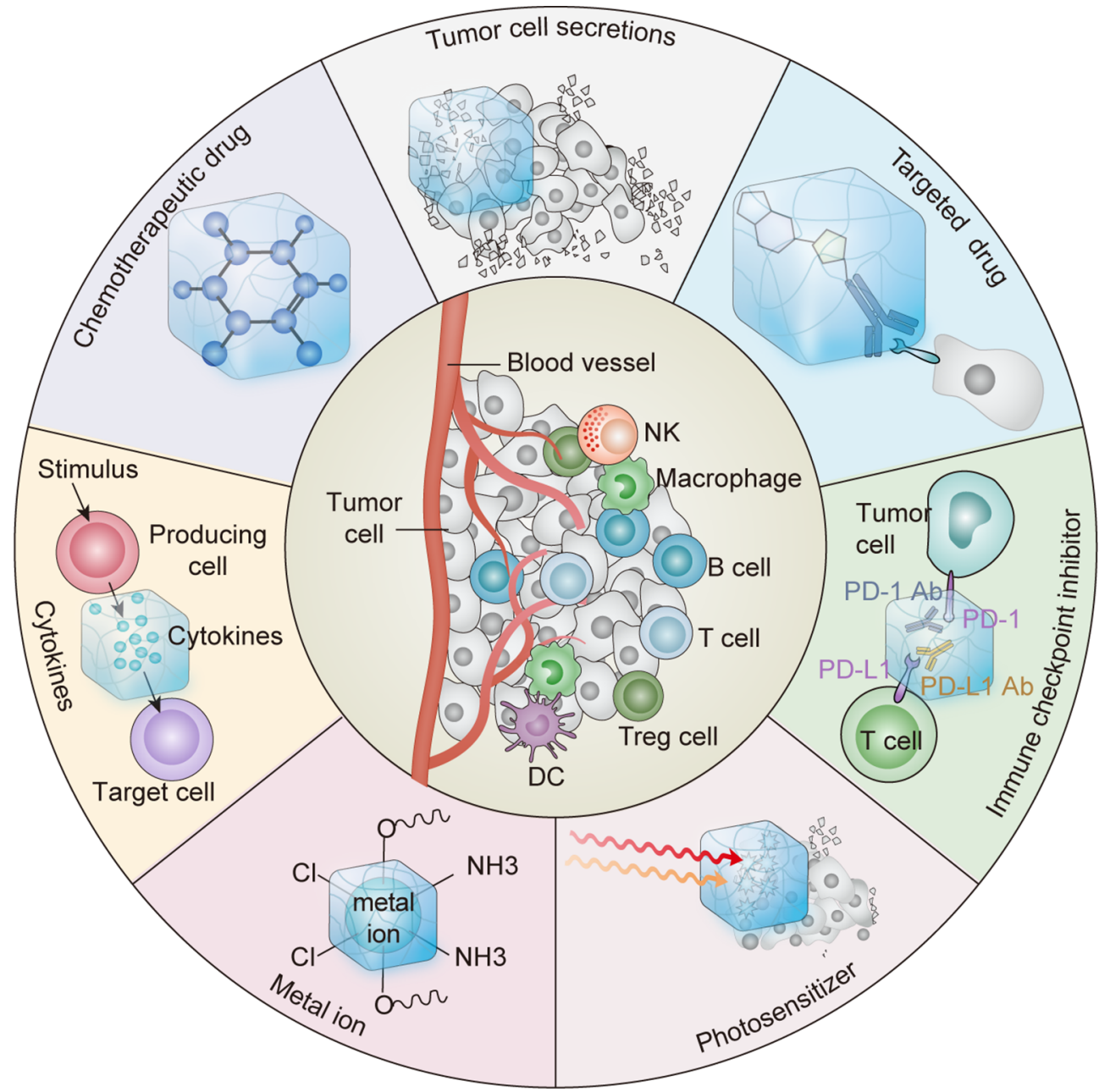

FIGURE 1 | Schematic diagram of antitumor therapy platform using hydrogels as platform to elicit antitumor immune response. 
composed of polysaccharides, proteins, and DNA. In contrast, synthetic hydrogels consist of biodegradable polymers, such as polypeptides and polyesters (7). Additionally, injectable hydrogels can be divided into ordinary hydrogels and smart hydrogels according to their responses to external stimuli. Ordinary injectable hydrogels are not sensitive to environmental changes, whereas smart injectable hydrogels can be affected by temperature, $\mathrm{pH}$, enzyme, and photoelectricity (13, 17). Moreover, injectable hydrogels can be biologically functionalized with targeting moieties that have an affinity for unique or overexpressed tumor cell markers for targeted drug delivery applications (18).

Over the past decade, many studies have investigated the antitumor potential of drug-loaded hydrogels (19). The therapeutic potential of hydrogels has also been investigated in patients with cancer. Up to September 2021, four clinical studies related to drug-loaded hydrogels for the treatment of cancer have been registered in the US registry of clinical trials (https:// clinicaltrials.gov/). Two completed, open-label, dose escalation clinical studies (NCT02891460, NCT02307487) evaluated the efficacy of mitomycin C-loaded hydrogels (TC-3) in patients with bladder cancer. The results of these studies have not been published yet (Table 1).

\section{IMMUNOSUPPRESSIVE STATUS OF TME}

TME is an integral part of tumors and can affect the efficacy of cancer treatment (9). At different stages of tumor development, different immune cell types are present in the TME. At an early stage, tumors are infiltrated by antitumor immune cells, including macrophages, natural killer (NK) cells, lymphocytes, and dendritic cells (DCs) (20). However, at later stages of tumor development, antitumor immune responses are hindered by immunosuppressive cells, such as myeloid-derived suppressor cells (MDSCs), regulatory T cells (Tregs), and M2 macrophages $(20,21)$. The balance between different types of immune cells determines the outcome of antitumor immune responses.

$\mathrm{CD}^{+}$cytotoxic $\mathrm{T}$ lymphocytes (CTLs) and $\mathrm{CD} 4^{+} \mathrm{T}$ helper (Th) cells are paramount immune cells for tumor cell elimination (22). Th1 responses, characterized by the production of IFN- $\gamma$, TNF- $\alpha$, and IL-2, are also essential for tumor rejection. However, Th1 responses can also contribute to tumor escape via IFN- $\gamma$ induced expression of the checkpoint molecule programmed death-ligand 1 (PD-L1) or tumor immunoediting and selection of resistant clones (23). In addition, long-term exposure of tumor antigens to Th1 cells and other T cell subtypes may promote the expression of inhibitory receptors, such as PD-L1, lymphocyte activation gene 3 protein (LAG-3), and T-cell immunoglobulin (Ig) domain and mucin domain protein 3 (TIM-3) (24). Immune checkpoint pathways in cancer cells can cause T-cell dysfunction and immune evasion. Immune checkpoint blockade (ICB), especially antibodies against cytotoxic T-lymphocyte-associated protein 4 (CTLA-4), programmed cell death protein 1 (PD-1), and PD-L1, can reverse immunosuppression and prevent immune evasion (9). ICB has shown remarkable long-term survival benefits in cancer patients with several types of tumors, including melanoma, non-small cell lung cancer, and renal cell carcinoma $(16,25)$.

However, Tregs, another subset of $\mathrm{CD}^{+}{ }^{+} \mathrm{T}$ cells, often inhibit antitumor immune responses and promote tumor growth. Tregs can directly interact with CTLs and NK cells or indirectly inhibit the antitumor activity of CTLs and NK cells by producing immunoregulatory cytokines, such as IL-10 and TGF- $\beta$ (10). Notably, Tregs have been associated with unfavorable survival in patients with many types of cancer (26). Hence, eliminating Tregs in the TME may enhance antitumor immune responses. Th2 cells can also block T-cell-induced tumor rejection by promoting $\mathrm{T}$-cell anergy, suppressing $\mathrm{T}$-cell-mediated cytotoxicity, and enhancing humoral immunity (10).

Tumor cells promote the recruitment of bone marrowderived cells (BMDCs), which can differentiate into tumorigenic cell subtypes under certain conditions (20). For instance, tumor-associated macrophages (TAMs) derived from BMDCs promote tumor progression by facilitating angiogenesis, invasion, and metastasis in vivo (27). MDSCs, another type of BMDCs, can suppress antitumor immune responses by inhibiting $\mathrm{T}$ cells and $\mathrm{NK}$ cells and promoting the expansion of Treg populations within the TME (21).

\section{INJECTABLE HYDROGELS TARGETING IMMUNOSUPPRESSIVE TUMOR MICROENVIRONMENT}

\section{Targeting Immune Checkpoint Molecules}

Immune checkpoint blockade (ICB) immunotherapies, especially antibodies against CTLA-4, PD-1, and PD-L1, have revolutionized cancer treatment (28). However, ICB monotherapies

TABLE 1 | Drugs embedded in hydrogels were used to treat cancers based clinical trials up to September 2021

\begin{tabular}{|c|c|c|c|}
\hline Study title & Conditions & Status & Identifier \\
\hline $\begin{array}{l}\text { A Prospective Open Label Comparative Dose Ranging Study Evaluating the Effect of Pre-TURBT Intravesical Instillation of } \\
\text { Mitomycin C (MMC) Mixed with TC-3 Gel in Patients with Non Muscle Invasive Bladder Cancer (NMIBC) }\end{array}$ & $\begin{array}{l}\text { Bladder } \\
\text { Cancer }\end{array}$ & Withdrawn & NCT01799499 \\
\hline $\begin{array}{l}\text { Safety and Tolerability Study Which Evaluate Intravesical Instillation with Mitomycin C Mixed with TC-3 Drug Retaining } \\
\text { Hydrogel Device in Patients with Muscle Invasive Bladder Cancer }\end{array}$ & $\begin{array}{l}\text { Bladder } \\
\text { Cancer }\end{array}$ & Completed & NCT02891460 \\
\hline Safety of Pre-TURBT Intravesical Instillation of Escalating Doses of TC-3 Gel and MMC in NMIBC Patients & $\begin{array}{l}\text { Bladder } \\
\text { Cancer }\end{array}$ & Completed & NCT02307487 \\
\hline Safety and Efficacy of Doxorubicin-eluting-bead Embolization in Patients with Advanced Hepatocellular Carcinoma & $\begin{array}{l}\text { Hepatocellular } \\
\text { Carcinoma }\end{array}$ & Unknown & NCT02525380 \\
\hline
\end{tabular}


show limited efficacy in most patients and may cause significant toxicity $(6,9,29)$. Therefore, more effective and safer combination therapies involving ICB are under development. PD-L1 expressed on the surface of tumor cells and on antigen-presenting cells can interact with PD-1 expressed on activated T cells, promoting T-cell apoptosis, anergy, and exhaustion $(30,31)$. Blocking the PD-1/PDL1 pathway with anti-PD-1 or anti-PD-L1 antibodies has demonstrated promising therapeutic efficacy in a variety of tumor types (32-35); however, response rates are only $10 \%-30 \%$ $(29,36)$. Low neoantigen burden, insufficient infiltration of tumorspecific T cells, and low expression of PD-L1 may contribute to the low response rates in cancer patients treated with ICB (20,37-41). Moreover, multiple administration cycles of anti-PD-1 antibodies can induce severe immune-related side effects (42-44); local delivery of antibodies can minimize off-target effects and increase drug bioavailability (45).

Wang et al. developed a drug-based supramolecular hydrogel for local delivery of immune checkpoint inhibitors (ICIs) to boost the host's immune system against tumors (Figure 2) (46). They first synthesized the amphiphilic prodrug, diCPTPLGLAG-iRGD, by conjugating a hydrophilic iRGD. This prodrug can spontaneously assemble into supramolecular nanotubes (P-NTs). By mixing a therapeutic dose of anti-PD-1 antibodies and P-NTs, they developed a hydrogel loaded with anti-PD-1 antibodies. Wang et al. found that this formulation could serve as a reservoir for long-term release of camptothecin (CPT) and anti-PD-1 antibodies within the TME, thereby inducing a potent antitumor immune response. They also found that local P-NT-anti-PD-1 treatment in GL-261 brain cancer and CT 26 colon cancer models led to tumor regression in $100 \%$ of mice.

The low immunogenicity of some tumor types and the body's decreased immune responses to tumor limit the development of immunotherapy. Immunogenic cell death (ICD), featured by the release of tumor-associated and tumor-specific antigens, dangerassociated molecular patterns, and pro-inflammatory cytokines, plays an essential role in cancer immunotherapy (47). Recent evidence suggests that neoadjuvant chemotherapy and the use of biomaterials-based delivery systems both enhance the therapeutic efficacy of immunotherapy owing to the induction of ICD $(48,49)$. Gu et al. engineered an injectable reactive oxygen species (ROS)-responsive hydrogel co-loaded with gemcitabine

A

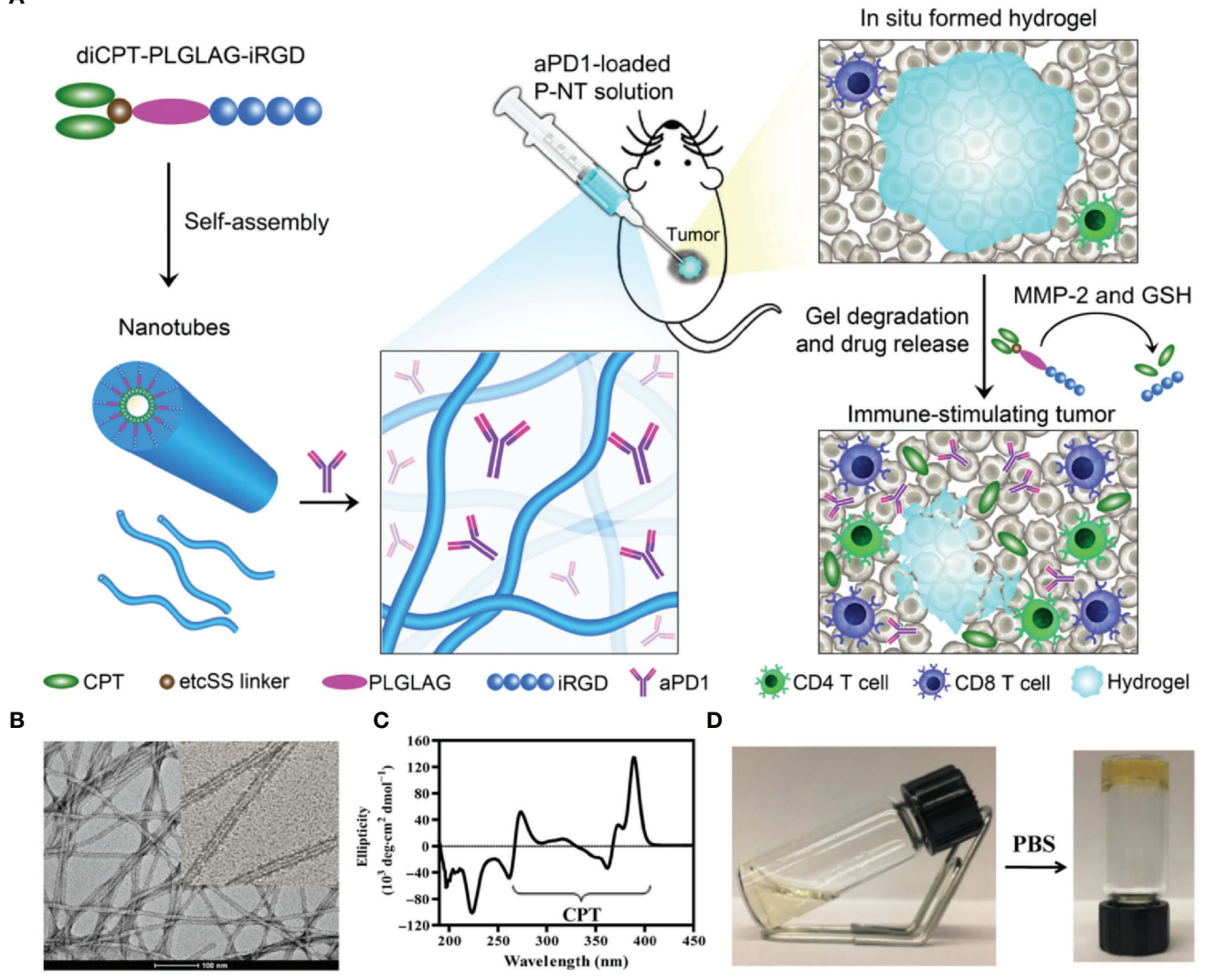

FIGURE 2 | Schematic illustration of the in situ formed P-NT-anti-PD-1 hydrogel. (A) In situ formation of P-NT-anti-PD-1 hydrogel, which enables localized CPT and anti-PD-1 delivery and promotes the activation of CD4 and CD8 T cells in the tumor microenvironment. (B) Representative transmission electron microscopy (TEM) image of the networks of the P-NT hydrogel. (C) Circular dichroism (CD) spectrum of camptothecin (CPT) solution. (D) Photographs of liquid P-NT transformed into hydrogel after the addition of phosphate-buffered saline (PBS). Reprinted with permission from Science Advances (46). 
(GEM) and anti-PD-L1 antibodies for in situ chemoimmunotherapy (50). As the scaffold consists of ROSdegradable hydrogel and the TME contains high levels of ROS, GEM and anti-PD-L1 antibodies can be specifically released in the TME. In B16-F10 melanoma and 4T1 breast tumor (lowimmunogenic) mouse models, local GEM delivery increased tumor immunogenicity and augmented the antitumor efficacy of ICB, thereby promoting tumor regression and suppressing tumor recurrence. To enhance the expression of tumorassociated antigens, Ruan et al. developed an in situ formed dual-bioresponsive gel depot for co-delivery of anti-PD-1 antibodies and zebularine (Zeb), a demethylation agent that enhances the expression of tumor-associated antigens (51). Anti-PD-1 antibodies were loaded into pH-sensitive $\mathrm{CaCO}_{3}$ nanoparticles (anti-PD1-NPs) and encapsulated with Zeb in the ROS-responsive hydrogel (Zeb-anti-PD-1-NPs-Gel). Local release of Zeb increased the immunogenicity of cancer cells and decreased immunosuppression. By doing so, Zeb boosted the ability of anti-PD-1 antibodies to induce $\mathrm{T}$ cell-mediated antitumor immune responses, inhibiting tumor growth and prolonging survival in mice bearing B16-F10 tumors. In addition to direct use of anti-PD-1 antibodies to block the PD1/PD-L1 pathway, targeting of a specific pathway that involves PD-L1 transcriptional repressors is also practicable. Li et al. reported a cancer cell membrane-derived hydrogel scaffold loaded with $\mathrm{Ca}^{2+}$ channel inhibitor dimethyl amiloride (DMA) and cyclin-dependent kinase 5 inhibitor roscovitine for cancer treatment. In this system, cancer cell membrane, DMA and roscovitine were chosen with the aim of creating an antigen depot, suppressing $\mathrm{Ca}^{2+}$-governed exosome secretion and downregulating tumor cell PD-L1 expression, respectively (52).

CTLA- 4 is expressed on activated Th1 cells and CTLs, and binds to co-stimulatory molecules CD80 and CD86 of antigenpresenting cells, thereby inhibiting the activation and proliferation of T cells (53). Although blocking CTLA-4 signaling unleashes antitumor immune responses, systemic administration of antiCTLA-4 antibodies may cause severe immune-related adverse events (5, 54-57). Chung et al. evaluated thermosensitive poloxamer 407 (P407) hydrogels as a slow-release system for optimizing anti-CTLA-4 therapy (58). They found that P407 hydrogel-mediated delivery of anti-CTLA-4 antibodies reduced serum antibody levels, mitigated the side effects of ICB, and exerted antitumor effects in mice bearing CT26 tumors. Similarly, Harui et al. found that local administration of hydrogel-encapsulated anti-CTLA-4 antibodies exhibited enhanced efficacy and minimal systemic toxicity in mice with MC-38 tumors (59). Peritumoral administration of $100 \mu \mathrm{g}$ of antiCTLA-4 antibodies loaded in hydrogels had similar or greater effects than systemic administration of $600 \mu \mathrm{g}$ of antibodies. While preserving antitumor activity, serum exposure following the administration of hydrogel-encapsulated anti-CTLA-4 was only $1 / 16$ th of that following systemic therapy.

Song et al. developed an injectable PEG-b-poly(L-alanine) (PEA) hydrogel to co-deliver a tumor vaccine consisting of tumor cell lysates (TCLs), granulocyte-macrophage colonystimulating factor (GM-CSF), and anti-CTLA-4 antibodies and
anti-PD-1 antibodies (60). TCLs, GM-CSF, anti-CTLA-4 antibodies, and anti-PD-1 antibodies were encapsulated into the porous PEA hydrogel by mixing these agents with PEA aqueous solution. Sustained release of tumor antigens and GMCSF promoted the recruitment and activation of DCs in vivo, inducing tumor-specific CTL responses. The extended release of ICIs from the hydrogel further enhanced T-cell activation and reduced Treg levels in the TME by blocking PD-1 and CTLA-4 pathways. Notably, the hydrogel-based combination therapy exhibited greater antitumor effects than the vaccine alone or ICB monotherapy in melanoma and 4T-1 mouse models.

\section{Targeting Tumor-Associated Macrophages}

Tumor-associated macrophages (TAMs) are a key component of the TME and play a significant role in tumor progression $(61,62)$. There are two main subtypes of TAMs: classically activated M1 macrophages (M1-TAMs) and alternatively activated M2 macrophages (M2-TAMs). M1-TAMs, which express high levels of IL-12 and IL-23, can scavenge foreign antigens and kill tumor cells (63). Tumor cells typically promote polarization of TAMs toward M2 in TME, facilitating IL-10 production and tumor growth (8). The balance between M1 and M2 TAMs has been associated with drug resistance, angiogenesis, and immunosuppression in tumors (8). Most macrophage-targeting therapies have three goals $(9,64)$ : (1) inhibit macrophage recruitment by blocking the $\mathrm{C}-\mathrm{C}$ motif chemokine ligand 2 (CCL2)/C-C motif chemokine receptor 2 (CCR2) axis $(65,66)$; (2) deplete macrophages or block -factor (CSF)-1/CSF-1R signaling $(67,68)$; (3) reprogram TAMs toward an M1-like phenotype using melittin (69), IFN- $\gamma$ (70), CD40 agonists (71), or tumor hypoxia-targeting agents (72). As macrophages are present throughout the body, systemic modulation of macrophages can lead to off-target effects and systemic toxicity (73). Furthermore, CCL2/CCR2- and CSF-1/CSF-1R-targeting strategies often result in the development of monocyte and macrophage populations that enhance neoangiogenesis and metastasis $(74,75)$.

M2-TAM depletion has proved effective in promoting tumor regression by suppressing TAM-associated immunosuppression (8). Although melittin is a potent anticancer agent, its hemolytic effects limit its clinical application. To overcome this obstacle, we developed a melittin-RADA32 hybrid peptide hydrogel. The melittin- and doxorubicin (DOX)-loaded peptide hydrogel (melittin-RADA32-DOX, or MRD hydrogel) exerted potent anti-melanoma effects by modulating the TME (76). Moreover, MRD hydrogels loaded with melittin and DOX exhibited direct cytotoxic effects, specifically depleted M2-like macrophages, and induced robust and long-lasting innate and adaptive immune responses. Notably, a single injection of the formulation significantly reduced the growth of primary melanoma tumors.

External stimuli can stimulate the reprogramming of M2TAMs into M1-TAMs, which have tumoricidal effects (77). KN93, a specific inhibitor of CAMKII, was found to have a direct tumoricidal activity and the ability to induce macrophage reprogramming (78). To further potentiate these effects of the 
melittin-RADA32 hydrogel, we designed a melittin-RADA24 peptide hydrogel loaded with KN93 (MR52-KN93; MRK hydrogel) (79). Compared with free KN93, the MRK hydrogel was more potent in eliminating tumor cells and inducing immunogenic cell death. Moreover, MRK significantly reduced the portion of M2-like TAMs and increased the ratio of M1-like to M2-like TAMs in the TME (Figure 3).

The TME is usually acidic due to the presence of hypoxia and glycolytic metabolism $(79,80)$. Cancer cell-derived lactate plays a critical role in the polarization of macrophages from the M1 phenotype to the M2 phenotype, which promotes tumor growth and metastasis (80). Liao et al. found that methylcellulose hydrogels loaded with lactate oxidase promoted lactate depletion and lactate-mediated repolarization of macrophages (81).

Several recent studies reported the direct involvement of TAMs in tumor resistance to ICB. By comparing the TME of ICB-resistant and ICB-sensitive murine tumors, Muraoka et al. found that TAMs in resistant tumors lacked antigen-presenting activity (82). They also found that cholesteryl-modified pullulan nanogels could efficiently deliver large peptides to TAMs and that upon TLR stimulation, the nanogel system elicited antigenpresenting activity in TAMs (82). By modulating TAMs, this

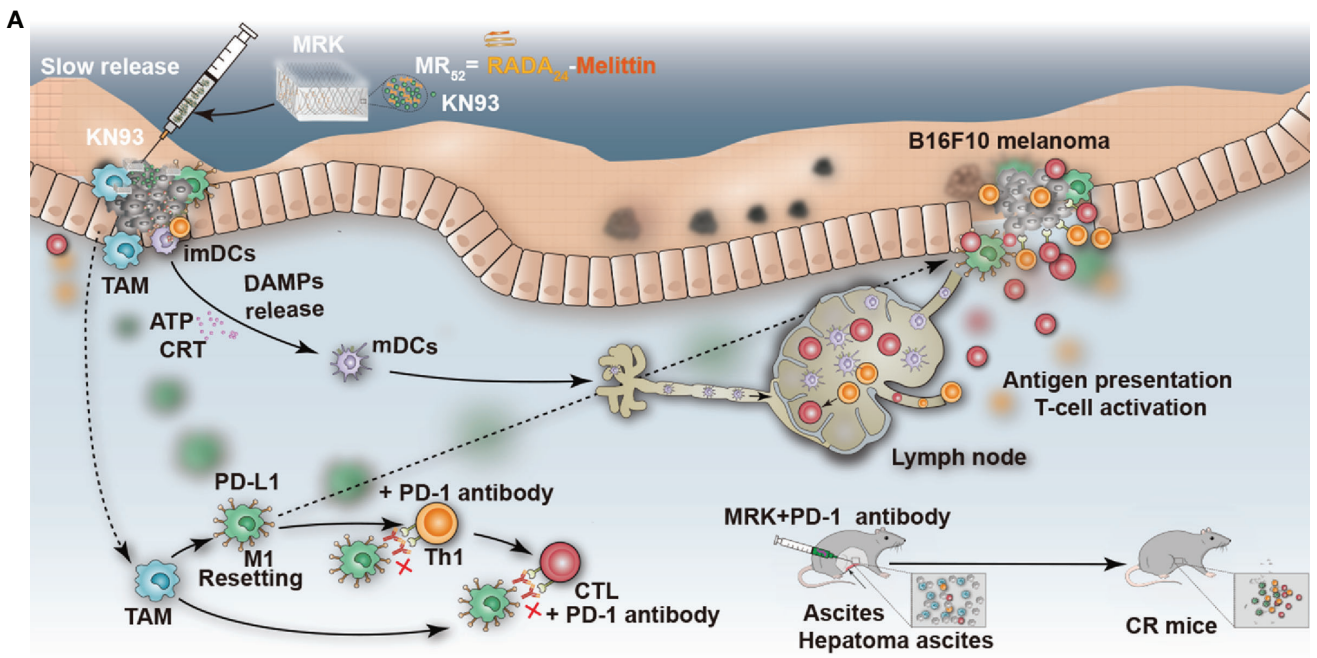

B
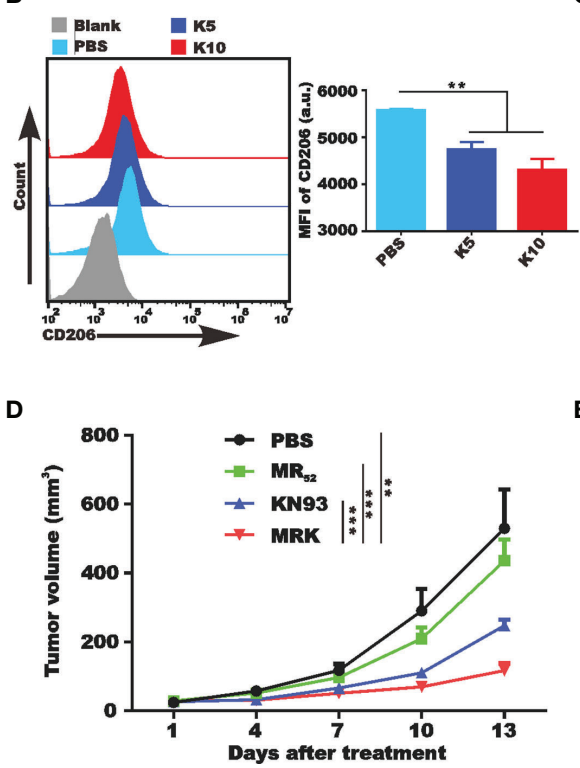

C
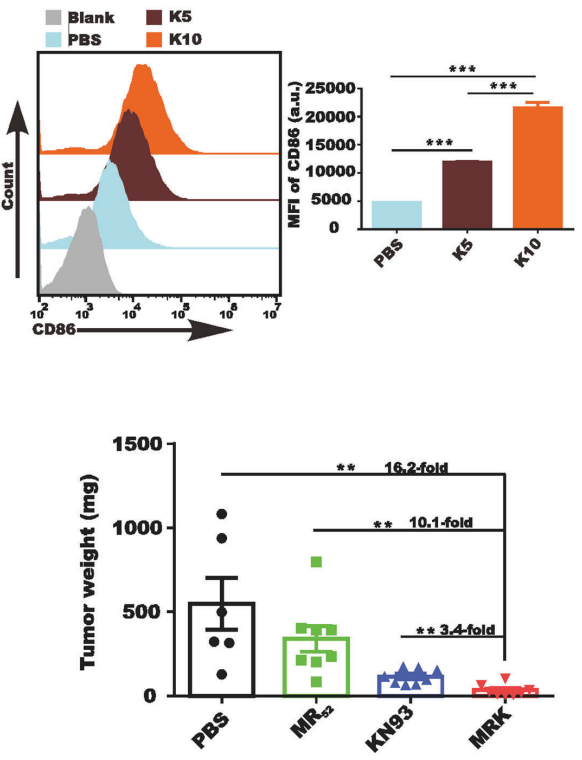

FIGURE 3 | In vivo activation of the immune system of tumor-bearing mice by MRK. (A) Schematic diagram summarizing the therapeutic effects of the MRK hydrogel alone or combined with anti-PD-1 antibodies. Subcutaneous injection of MRK stimulates dendritic cell maturation and T cell activation in the lymph nodes. Activated T cells eliminate tumor cells. MRK can also stimulate M1-type polarization of tumor-associated macrophages, activating Th1 cells and cytotoxic $T$ lymphocytes. MRK combined with PD-1 alleviates hepatocellular ascites in mice. (B, C) Comparison of the production of M2-type macrophages (B) and dendritic cells (C) in each group. (D, E) Tumor volume (D) and weight (E) in different groups. Reprinted with permission from Theranostics $(78) .{ }^{\star \star} \mathrm{P}<0.01,{ }^{\star \star \star} \mathrm{P}<0.001$. 
formulation transformed ICB-resistant tumors into ICBsensitive tumors. These results strongly support targeting TAMs as a promising strategy for enhancing the efficacy of cancer immunotherapy.

Because M1-TAMs can promote tumor rejection, direct injection of M1-TAMs can significantly cause tumor regression in vivo; however, the induction of acute inflammatory responses limits the clinical translation of this approach (83). To improve this strategy, Guerra et al. employed a synthetic extracellular matrix (ECM) system consisting of cross-linked PEGdA and Gel-PEG-Cys as a carrier for local delivery of activated M1 macrophages. They found that M1-loaded hydrogels promoted apoptosis in hepatocellular carcinoma cells and tumor regression in vivo while exhibiting low immunogenicity, high biocompatibility, and improved release kinetics (84).

\section{Targeting the Tumor Vasculature}

Normal vascularization is critical for nutrients and oxygen supply, as well as metabolic waste removal. However, abnormal vascularization characterized by immature, disorganized, and permeable blood vessels creates a hostile TME characterized by hypoxia, low $\mathrm{pH}$, low interstitial fluid pressure, decreased immune cell infiltration and activity, and increased risk of metastasis $(85,86)$. Furthermore, abnormal vascularization reduces the diffusion of chemotherapeutic drugs and impairs the efficacy of radiotherapy (86). Therefore, vascular normalization could restore tumor perfusion and oxygenation and enhance the efficacy of chemotherapy and radiotherapy $(87,88)$.

Antibodies against vascular endothelial growth factor (VEGF) have emerged as a promising therapeutic strategy for solid tumors, as tumor growth and metastasis require neoangiogenesis (89). Targeting VEGF signaling induces tumor vasculature normalization, further reprogramming the immunosuppressive TME and increasing the number of tumor-infiltrating lymphocytes (TILs) $(90,91)$. Bevacizumab, the first approved anti-VEGF drug to inhibit tumor angiogenesis in the United States, has a limited half-life and membrane permeability. To overcome these limitations, Ferreira and coworkers designed a bevacizumab-loaded alginate hydrogel for localized anti-VEGF cancer therapy by mixing alginate solution with bevacizumab and cross-linking it with calcium chloride (92). The tridimensional hydrogel increased drug stability, especially in acid environments, and provided slow and continuous drug release to the tumor and surrounding tissues after local application. Moreover, with the development of photodynamic therapy (PDT), it has shown the potential to trigger local and systemic antitumor immune responses. However, abnormal angiogenesis and hypoxia in TME promote immunosuppression. The immune response after routine PDT is usually insufficient to cause tumor regression, which limits the efficacy of PDT. Based on this, Zhou et al. developed a prolonged oxygen-generating phototherapy hydrogel (POP-Gel) system by combining the photosensitizerloaded thermosensitive hydrogel with calcium superoxide and catalase to relieve tumor hypoxia. Long-term effective oxygen supply improved the hypoxic state of TME and down-regulated the expression of HIF- $1 \alpha$ and VEGF, further inducing a robust antitumor adaptive immune response (93).

RNA interference (RNAi) enables robust and specific gene silencing, providing a promising therapeutic avenue for cancer treatment. However, efficient drug delivery systems for short interfering RNAs (siRNAs) are lacking (94-96). Fujii et al. developed a self-assembled nanogel of cholesterol-bearing cycloamylose with a spermine group ( $\mathrm{CH}-\mathrm{CA}-\mathrm{Spe})$ as a carrier to deliver VEGF-specific siRNAs (siVEGFs) into tumor cells. This system showed low toxicity in patients, efficient intratumor delivery, and high stability in vivo (97). The siVEGF-nanogel complex was taken up by tumor cells via the lysosomal pathway and suppressed VEGF expression in renal cell carcinoma cells. Intratumoral injections of the complex effectively suppressed tumor growth and neovascularization. The treatment also significantly suppressed MDSC infiltration and IL-17A production in the spleen, suggesting that silencing of VEGF locally in the tumor may modulate systemic immune responses.

Despite promising findings in preclinical models, the efficacy of anti-angiogenic therapies in the clinic has been disappointing, as most patients exhibit innate or acquired resistance to the treatment (98). However, anti-angiogenic therapeutics can increase the efficacy of immunotherapy (99). Additionally, low doses of anti-VEGF antibodies can induce vascular normalization, prevent the differentiation of TAMs toward an immune inhibitory M2-like phenotype, and block VEGFmediated inhibition of DC maturation (90). Therefore, vascular normalization with anti-angiogenic therapies in combination with other therapies may be an attractive therapeutic strategy. Pal et al. developed a biocompatible self-assembled lithocholic acid dipeptide-derived hydrogel (TRI-Gel), which provided sustained delivery of DOX, anti-angiogenic combretastatin-A4 (CA4), and dexamethasone (100). TRI-Gel therapy inhibited cancer cell proliferation, angiogenesis, and inflammation at the tumor site, thereby suppressing tumor progression and prolonging median survival with reduced drug resistance (100). Yu et al. designed an in situ thermo-gelling hydrogel (mPEG-bPELG) to co-deliver combretastatin A4 disodium phosphate (CA4P) and cisplatin (CDDP) for the local treatment of colon cancer (101). Compared with the free drugs, the CA4P and CDDP co-loaded gel induced less tumor cell death in vitro, while its antitumor effect was highest in C26 tumor-bearing mice after peritumoral injection (101).

Starvation therapies can inhibit tumor progression by decreasing nutrient supply indispensable for tumor growth $(102,103)$. Blood vessel occlusion can permanently occlude blood and nutrition supply to the tumor. However, this strategy is often associated with poor persistence, frequent tumor metastasis and recurrence, and embolism in normal blood vessels. Zhang and coworkers established an extravascular gelation shrinkage-derived internal stress strategy to narrow blood vessels, occlude blood and nutrition supply, reduce vascular density, induce hypoxia and apoptosis, and ultimately promote starvation of the tumor (104). To this end, they engineered an organic-inorganic composite hydrogel 
consisting of PEG-SH-modified gold nanorods (GNR-PEG-SH) and thermal-sensitive hydrogel mixture (chitosan (CS)/mPEG$\mathrm{Mal} / \mathrm{pNIPAAm-co-AAc}$; hydrogel-GNR). When irradiated with an $808 \mathrm{~nm}$ laser, hydrogel-GNR induced internal stress, which narrowed intratumor and adjoining blood vessels in a GNRdependent manner. This starvation therapy inhibited tumor progression in both PANC-1 pancreatic cancer and 4T1 breast cancer mouse models. Importantly, this starvation strategy suppressed tumor metastasis and tumor recurrence by reducing vascular density, occluding blood and nutrition supply (Figure 4).

\section{Targeting Other Immunoregulatory Cells and Factors}

In view of the strong immunosuppressive effect of Tregs in the TME, targeting Tregs has emerged as an attractive strategy to unleash antitumor immune responses and reenforce immune-mediated tumor rejection (10). Tumor-specific Tregs residing at the TME express high levels of CTLA-4 and OX40, and in situ injection of anti-CTLA-4 and anti-OX40 together with CpG can deplete tumor-infiltrating Tregs (104). This in situ immunomodulation approach activates systemic antitumor immune responses more effectively than systemic immunomodulation strategies (105). The co-delivery of tumor anti-CTLA-4, anti-PD-1, and tumor vaccines using injectable PEG-b-poly(L-alanine) hydrogels increased the efficacy of immunotherapy by reducing the number of Tregs and increasing the number of activated $\mathrm{CD}^{+} \mathrm{T}$ cells in the TME (60). In addition to directly killing tumor cells, some chemotherapeutic agents can regulate the immune system through various mechanisms, including the modulation of Tregs (106-112). Co-delivery of DOX and CpG self-crosslinking nanoparticles (CpG NPs) using injectable $\alpha$-cyclodextrin/polyethylene glycol hydrogels increased the number of cytotoxic $\mathrm{CD}^{+} \mathrm{T}$ lymphocytes and decreased the numbers of MDSCs, M2-TAMs, and Tregs in the TME (107). Additionally, although chemotherapy alone reduced the number of Tregs to some extent, combination therapy using $\alpha$-cyclodextrin/
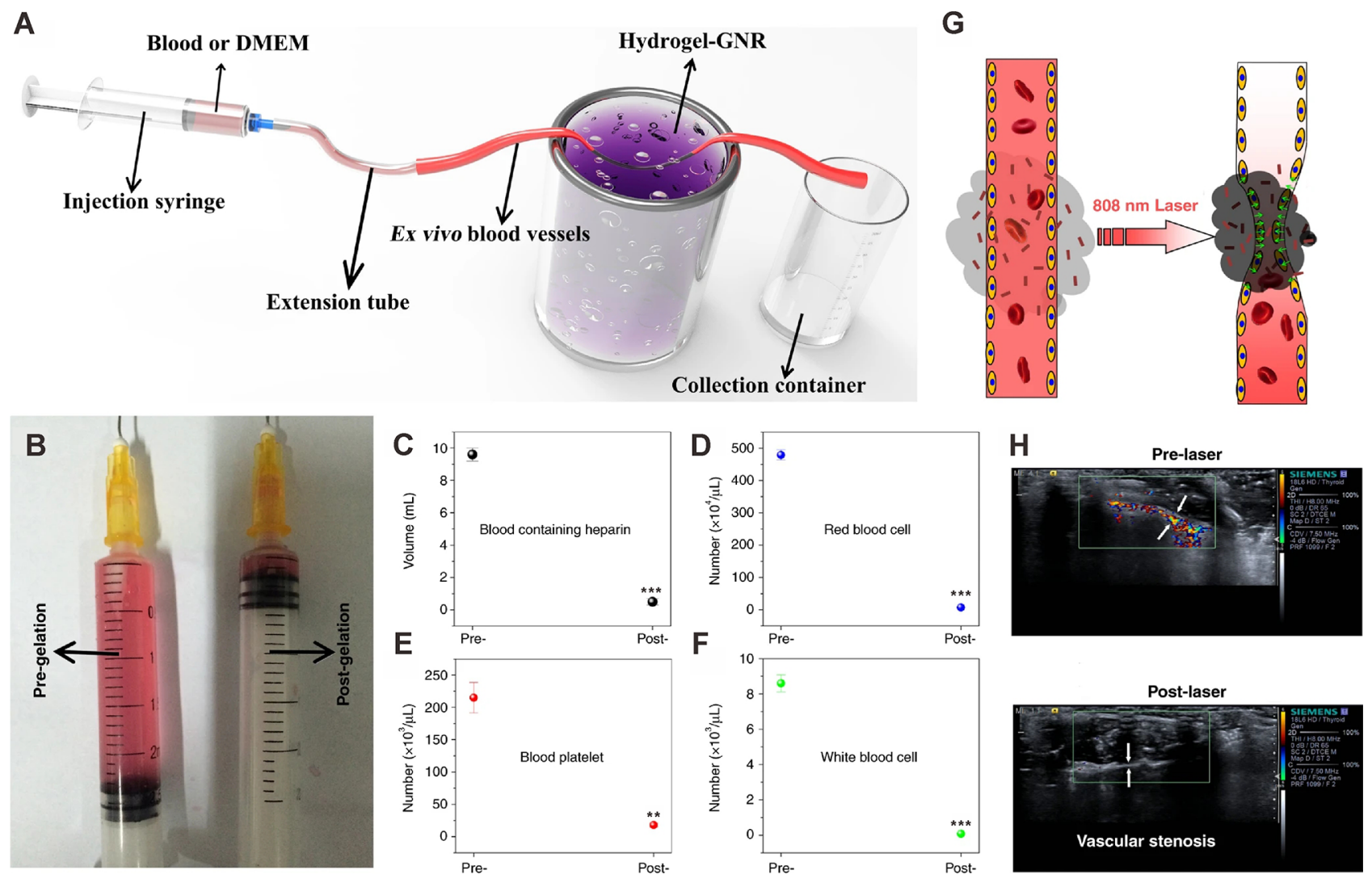

FIGURE 4 | Occlusion of blood supply in ex vivo and in vivo artery models. (A) Schematic of the experimental apparatus for evaluating vessel occlusion ex vivo in blood vessels (with an inner diameter of $1.00 \mathrm{~mm}$ ) treated with the gelation shrinkage-induced internal stress platform. (B) Traversed volume of DMEM in the ex vivo blood vessel model before (left) and after (right) gelation of hydrogel-GNR. (C-F) Collected blood volume (C), red blood cells (D), blood platelet (E), and white blood cells $(\mathbf{F})$ traversed through ex vivo blood vessels using rabbit blood containing heparin. ${ }^{* \star} \mathrm{P}<0.01$ and ${ }^{* \star *} \mathrm{P}<0.001$ compared to pre-gelation; determined using Student's t-test. (G) Schematic representation of the extravascular gelation shrinkage-induced internal stress system irradiated with 808 nm laser. (H) CDFI images of abdominal arteries of nude mice treated which hydrogel-GNR. CDFI images were captured before and after irradiation with an $808 \mathrm{~nm}$ laser. White arrows indicate the blood vessels of the abdominal artery. Reprinted with permission from Springer Nature (104). 
polyethylene glycol hydrogels-CpG NP-DOX remarkably reduced the number of Tregs in the TME (107).

The balance between different immune cell subsets, immune factors, and signaling molecules determine the outcome of antitumor immune response. Intratumoral delivery of immunomodulatory cytokines has been tested in the clinic as a strategy to augment antitumor immune responses (10). To elicit a therapeutic response, sufficient concentrations and long-lasting release of cytokines in TME are necessary, along with a non-toxic concentration of the cytokine outside of TME. GM-CSF, IL-2, IL-12, and IFN- $\gamma$ are among the several cytokines tested for local cancer treatment based on injectable hydrogels (16). Son et al. demonstrated that GM-CSF improved the function of antigenpresenting cells and enhanced antitumor immune responses (113). Co-delivery of GM-CSF and anticancer drugs using a chitosan-based hydrogel system resulted in a synergistic anticancer effect, as tumor-specific $\mathrm{CD} 8^{+} \mathrm{T}$ cell responses were significantly enhanced (113). Den Otter et al. developed physically crosslinked dextran hydrogels for the local delivery of IL2. The system exhibited a strong therapeutic effect, enhancing the clinical applicability of IL-2 (114). Kurisawa and coworkers developed an injectable hyaluronic acidtyramine (HATyr) conjugate hydrogel to locally deliver IFN- $\alpha 2$ a to treat liver cancer (115). The enzymatically crosslinked HATyr hydrogel released IFN- $\alpha 2 \mathrm{a}$ in the TME and inhibited tumor growth while providing tunable hydrogel stiffness and rapid gelation rate (115). Eonju $\mathrm{Oh}$ et al. utilized gelatin-based hydrogels for sustained co-delivery of DCs and oncolytic adenovirus (oAd) co-expressing IL-12 and GM-CSF while preserving the biological activity of the cytokines (116). Compared with single treatment (oAd or DC) or combination treatment without the gel $(o A d+D C)$, oAd+DC/gel treatment resulted in a significantly higher expression of IL-12, GM-CSF, and IFN- $\gamma$ in tumors through a positive feedback loop. The high levels of IL-12, GM-CSF, and IFN- $\gamma$ in the TME strongly activated endogenous and exogenous DCs, which migrated to the draining lymph nodes and promoted the activation and infiltration of $\mathrm{CD} 4^{+}$ and $\mathrm{CD}^{+} \mathrm{T}$ cells into the tumor, finally leading to robust tumor regression. Interestingly, oAd+DC/gel treatment also alleviated tumor-induced thymic atrophy (Figure 5).

Chronic inflammation in TME can promote cancer progression in several ways, and remission of chronic inflammation can help control the tumor (117). The cyclooxygenase 2 (COX2) inhibitor celecoxib has been shown to exert antitumor effects in various human cancers $(118,119)$. For instance, simultaneous and local administration of anti-PD-1 monoclonal antibodies and celecoxib using alginate hydrogels resulted in stronger antitumor effects than anti-PD-1 or celecoxib alone. In addition, the formulation elicited a potent and sustained antitumor immune response (120). Notably, codelivery of celecoxib and anti-PD-1 monoclonal antibodies increased the numbers of INF- $\gamma$-expressing $\mathrm{CD} 4^{+}$and $\mathrm{CD} 8^{+} \mathrm{T}$ cells and decreased the numbers of intratumoral Tregs, MDSCs, and PD-L1-positive tumor cells. Furthermore, this co-delivery system enhanced the expression of the anti-angiogenic chemokines CXCL9 and CXCL10 and suppressed the intratumoral production of IL-1, IL-6, and COX2, suggesting reduced inflammation and angiogenesis in the tumor.

\section{CONCLUSION}

Numerous injectable hydrogels have been developed over the past years (121). Injectable hydrogels offer many advantages, including good biocompatibility and biodegradability, minimal invasion, convenient synthesis, versatility, high drug-loading capacity, and controlled drug release ability (122). Owing to their unique properties, injectable hydrogels can be used as drug delivery systems, which can locally and continuously release therapeutic agents. Although intratumoral injections suffer from localized treatment and inhomogenous distribution across tumors, injectable hydrogels as drug delivery systems can overcome many limitations of current systemic therapies for cancer, especially systemic toxicity and limited efficacy (123). Compared with intravenous delivery, the intratumoral injection can provide direct contact with tumor cells and immune cells, eliciting a more strong and long-lasting immune response. Besides localized treatment for single tumor, injectable hydrogels can be applied for the treatment of extensive pleural and peritoneal metastasis, such as malignant pleural effusion and malignant ascites. More importantly, in some cases, injectable hydrogels can not only effectively promote ICD of tumor cells and reshape immunosuppressive TME against local tumors but also often generate abscopal effect against distant metastases by activating systemic antitumor immunity (124).

To eradicate cancer cells, effector immune cells must first be activated and overcome the multiple suppressive factors in the TME. Strategies to reverse the immunosuppressive TME include the targeted inhibition of key immunomodulatory factors in the TME using inhibitors of angiogenesis (89), ICIs (60), and agents targeting immunoregulatory cells and factors (113). Off-target effect and treatment resistance greatly weaken the therapeutic effect of single treatment regimen. Therefore, a shift from monotherapy to combination therapies is essential to provide more options of available treatments. The development of novel combination therapies may help enhance the antitumor effects of current therapies and prevent the development of treatment resistance. Hydrogels provide a promising platform for the codelivery of multiple agents targeting various components of the TME while causing minimal systemic toxicity. In addition, injectable hydrogels can also be combined with conventional treatments, such as radiotherapy and chemotherapy, to transform immunosuppressive TME to a pro-inflammatory state and amplify the antitumor immune response (50, 121, 125).

Despite the advances in injectable hydrogels, there are still several challenges that limit their clinical translation. It is necessary to determine at which stage of tumorigenesis a given treatment is most effective, and whether the effect of treatments depends on the composition of TME at the primary and metastatic sites. Although several combination systems demonstrate synergistic effects, their compositions need to be further optimized to maximize their 
A

GHPA conjugates
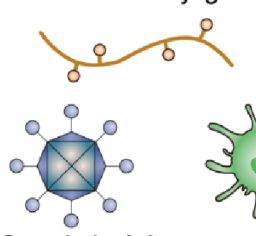

Oncolytic Ad Dendritic cell

expressing IL-12 and GM-CSF
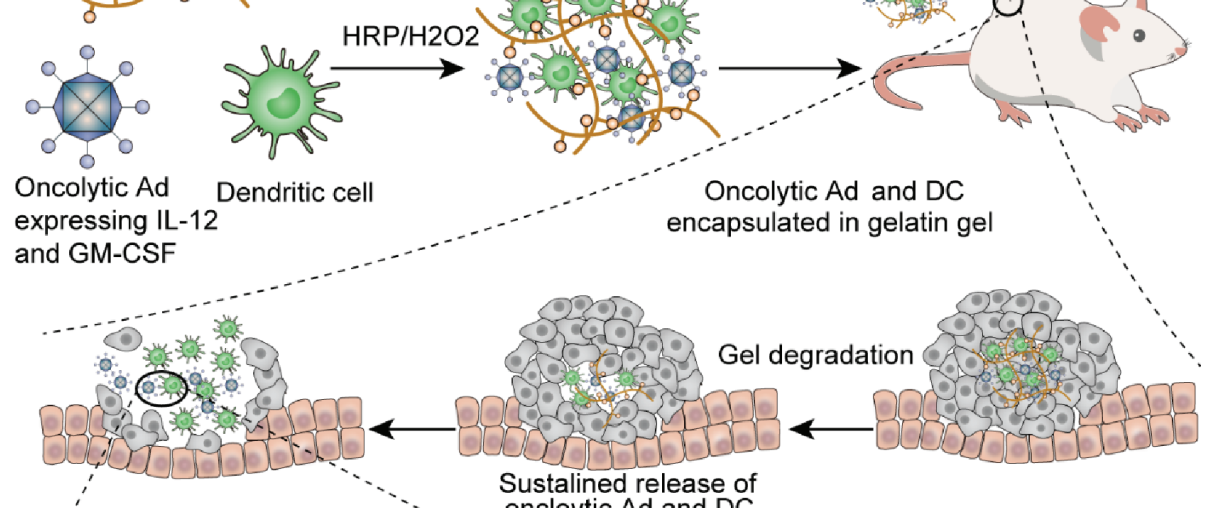

Oncolytic Ad and DC encapsulated in gelatin gel
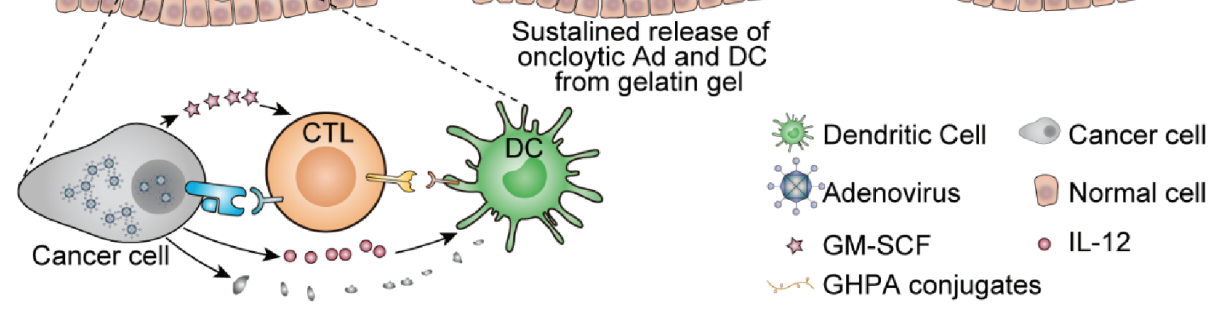

B

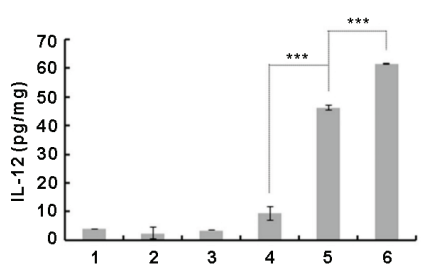

C
D

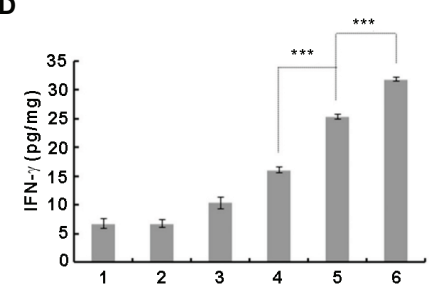

FIGURE 5 | Oncolytic adenoviruses and dendritic cells encapsulated in gelatin gels activate the immune system to eliminate tumor cells and induce the expression of IL-12 and GM-CSF in tumor cells. (A) Schematic representation of optimized biodegradable polymeric reservoir-mediated local and sustained co-delivery of dendritic cells and oncolytic adenovirus expressing IL-12 and GM-CSF. (B-D) The expression levels of IL-12 (B), GM-CSF (C), and IFN- $\gamma$ (D) in tumors. Reprinted with permission from Elsevier (116). ${ }^{* *} \mathrm{P}<0.001$.

antitumor efficacy and reduce side effects. Furthermore, future work is required to ensure that in addition to exerting antitumor effects locally and modulating the TME, hydrogels also activate systemic immune responses to prevent metastasis and tumor recurrence. Future multidisciplinary studies are warranted to design injectable hydrogel-based delivery systems for the codelivery and sequential release of different therapeutic agents to maximize the overall therapeutic efficiency of cancer therapies and accelerate their clinical translation, especially in some late-stage cancers, such as malignant pleural effusion and malignant ascites (126).

\section{AUTHOR CONTRIBUTIONS}

YL and YG wrote the manuscript. JH and HJ drafted the outline for the review and revised the manuscript. BY and P-CL checked the format and content of the manuscript. All authors contributed to the article and approved the submitted version.

\section{FUNDING}

This work was supported by National Natural Science Foundation of China (Grant No. 82022040 and 81874233).

2. Couzin-Frankel J. Breakthrough of the Year 2013. Cancer Immunotherapy. Science (2013) 342:1432-3. doi: 10.1126/science.342.6165.1432

3. Mellman I, Coukos G, Dranoff G. Cancer Immunotherapy Comes of Age. Nature (2011) 480:480-9. doi: 10.1038/nature10673
1. Siegel RL, Miller KD, Jemal A. Cancer Statistics, 2017. CA Cancer J Clin (2017) 67:7-30. doi: 10.3322/caac.21387 
4. Sang W, Zhang Z, Dai Y, Chen X. Recent Advances in Nanomaterial-Based Synergistic Combination Cancer Immunotherapy. Chem Soc Rev (2019) 48:3771-810. doi: 10.1039/c8cs00896e

5. Michot JM, Bigenwald C, Champiat S, Collins M, Carbonnel F, Postel-Vinay $\mathrm{S}$, et al. Immune-Related Adverse Events With Immune Checkpoint Blockade: A Comprehensive Review. Eur J Cancer (2016) 54:139-48. doi: 10.1016/j.ejca.2015.11.016

6. Milling L, Zhang Y, Irvine DJ. Delivering Safer Immunotherapies for Cancer. Adv Drug Deliv Rev (2017) 114:79-101. doi: 10.1016/ j.addr.2017.05.011

7. Chao Y, Chen Q, Liu Z. Smart Injectable Hydrogels for Cancer Immunotherapy. Adv Funct Mater (2020) 30:ARTN 1902785. doi: 10.1002/adfm.201902785

8. Musetti S, Huang L. Nanoparticle-Mediated Remodeling of the Tumor Microenvironment to Enhance Immunotherapy. ACS Nano (2018) 12:11740-55. doi: 10.1021/acsnano.8b05893

9. Phuengkham H, Ren L, Shin IW, Lim YT. Nanoengineered Immune Niches for Reprogramming the Immunosuppressive Tumor Microenvironment and Enhancing Cancer Immunotherapy. Adv Mater (2019) 31:e1803322. doi: 10.1002/adma.201803322

10. Pitt JM, Marabelle A, Eggermont A, Soria JC, Kroemer G, Zitvogel L. Targeting the Tumor Microenvironment: Removing Obstruction to Anticancer Immune Responses and Immunotherapy. Ann Oncol (2016) 27:1482-92. doi: 10.1093/annonc/mdw168

11. Smyth MJ, Ngiow SF, Ribas A, Teng MW. Combination Cancer Immunotherapies Tailored to the Tumour Microenvironment. Nat Rev Clin Oncol (2016) 13:143-58. doi: 10.1038/nrclinonc.2015.209

12. Li Y, Rodrigues J, Tomas H. Injectable and Biodegradable Hydrogels: Gelation, Biodegradation and Biomedical Applications. Chem Soc Rev (2012) 41:2193-221. doi: 10.1039/c1cs15203c

13. Norouzi M, Nazari B, Miller DW. Injectable Hydrogel-Based Drug Delivery Systems for Local Cancer Therapy. Drug Discov Today (2016) 21:1835-49. doi: 10.1016/j.drudis.2016.07.006

14. Oliva N, Conde J, Wang K, Artzi N. Designing Hydrogels for On-Demand Therapy. Acc Chem Res (2017) 50:669-79. doi: 10.1021/acs.accounts. $6 \mathrm{~b} 00536$

15. Seliktar D. Designing Cell-Compatible Hydrogels for Biomedical Applications. Science (2012) 336:1124-8. doi: 10.1126/science.1214804

16. Yu S, He C, Chen X. Injectable Hydrogels as Unique Platforms for Local Chemotherapeutics-Based Combination Antitumor Therapy. Macromol Biosci (2018) 18:e1800240. doi: 10.1002/mabi.201800240

17. Fan DY, Tian Y, Liu ZJ. Injectable Hydrogels for Localized Cancer Therapy. Front Chem (2019) 7:675. doi: 10.3389/fchem.2019.00675

18. Shu C, Li R, Yin Y, Yin D, Gu Y, Ding L, et al. Synergistic Dual-Targeting Hydrogel Improves Targeting and Anticancer Effect of Taxol In Vitro and In Vivo. Chem Commun (Camb) (2014) 50:15423-6. doi: 10.1039/c4cc05614k

19. Fathi M, Alami-Milani M, Geranmayeh MH, Barar J, Erfan-Niya H, Omidi Y. Dual Thermo-and pH-Sensitive Injectable Hydrogels of Chitosan/(Poly (N-Isopropylacrylamide-Co-Itaconic Acid)) for Doxorubicin Delivery in Breast Cancer. Int J Biol Macromol (2019) 128:957-64. doi: 10.1016/ j.ijbiomac.2019.01.122

20. Gajewski TF, Schreiber H, Fu YX. Innate and Adaptive Immune Cells in the Tumor Microenvironment. Nat Immunol (2013) 14:1014-22. doi: 10.1038/ ni. 2703

21. Marvel D, Gabrilovich DI. Myeloid-Derived Suppressor Cells in the Tumor Microenvironment: Expect the Unexpected. J Clin Invest (2015) 125:335664. doi: 10.1172/JCI80005

22. Fridman WH, Pages F, Sautes-Fridman C, Galon J. The Immune Contexture in Human Tumours: Impact on Clinical Outcome. Nat Rev Cancer (2012) 12:298-306. doi: 10.1038/nrc3245

23. Schreiber RD, Old LJ, Smyth MJ. Cancer Immunoediting: Integrating Immunity's Roles in Cancer Suppression and Promotion. Science (2011) 331:1565-70. doi: 10.1126/science.1203486

24. Speiser DE, Utzschneider DT, Oberle SG, Munz C, Romero P, Zehn D. T Cell Differentiation in Chronic Infection and Cancer: Functional Adaptation or Exhaustion? Nat Rev Immunol (2014) 14:768-74. doi: 10.1038/nri3740

25. Ribas A, Wolchok JD. Cancer Immunotherapy Using Checkpoint Blockade. Science (2018) 359:1350-5. doi: 10.1126/science.aar4060
26. Shang B, Liu Y, Jiang SJ, Liu Y. Prognostic Value of Tumor-Infiltrating FoxP3+ Regulatory T Cells in Cancers: A Systematic Review and MetaAnalysis. Sci Rep (2015) 5:15179. doi: 10.1038/srep15179

27. Joyce JA, Pollard JW. Microenvironmental Regulation of Metastasis. Nat Rev Cancer (2009) 9:239-52. doi: 10.1038/nrc2618

28. Sharma P, Allison JP. The Future of Immune Checkpoint Therapy. Science (2015) 348:56-61. doi: 10.1126/science.aaa8172

29. Kuai R, Yuan W, Son S, Nam J, Xu Y, Fan Y, et al. Elimination of Established Tumors With Nanodisc-Based Combination Chemoimmunotherapy. Sci $A d v$ (2018) 4:eaao1736. doi: 10.1126/sciadv.aao1736

30. Boussiotis VA. Molecular and Biochemical Aspects of the PD-1 Checkpoint Pathway. N Engl J Med (2016) 375:1767-78. doi: 10.1056/NEJMra1514296

31. Zou W, Wolchok JD, Chen L. PD-L1 (B7-H1) and PD-1 Pathway Blockade for Cancer Therapy: Mechanisms, Response Biomarkers, and Combinations. Sci Transl Med (2016) 8:328rv4. doi: 10.1126/scitranslmed.aad7118

32. Brahmer JR, Tykodi SS, Chow LQ, Hwu WJ, Topalian SL, Hwu P, et al. Safety and Activity of Anti-PD-L1 Antibody in Patients With Advanced Cancer. N Engl J Med (2012) 366:2455-65. doi: 10.1056/NEJMoa1200694

33. Hamid O, Robert C, Daud A, Hodi FS, Hwu WJ, Kefford R, et al. Safety and Tumor Responses With Lambrolizumab (Anti-PD-1) in Melanoma. N Engl J Med (2013) 369:134-44. doi: 10.1056/NEJMoa1305133

34. Powles T, Eder JP, Fine GD, Braiteh FS, Loriot Y, Cruz C, et al. MPDL3280A (Anti-PD-L1) Treatment Leads to Clinical Activity in Metastatic Bladder Cancer. Nature (2014) 515:558-62. doi: 10.1038/nature13904

35. Robert C, Long GV, Brady B, Dutriaux C, Maio M, Mortier L, et al. Nivolumab in Previously Untreated Melanoma Without BRAF Mutation. N Engl J Med (2015) 372:320-30. doi: 10.1056/NEJMoa1412082

36. Robert C, Schachter J, Long GV, Arance A, Grob JJ, Mortier L, et al. Pembrolizumab Versus Ipilimumab in Advanced Melanoma. $N$ Engl J Med (2015) 372:2521-32. doi: 10.1056/NEJMoa1503093

37. Gibney GT, Weiner LM, Atkins MB. Predictive Biomarkers for Checkpoint Inhibitor-Based Immunotherapy. Lancet Oncol (2016) 17:e542-51. doi: 10.1016/S1470-2045(16)30406-5

38. Hegde PS, Karanikas V, Evers S. The Where, the When, and the How of Immune Monitoring for Cancer Immunotherapies in the Era of Checkpoint Inhibition. Clin Cancer Res (2016) 22:1865-74. doi: 10.1158/1078-0432.CCR-15-1507

39. Schumacher TN, Schreiber RD. Neoantigens in Cancer Immunotherapy. Science (2015) 348:69-74. doi: 10.1126/science.aaa4971

40. Tang H, Wang Y, Chlewicki LK, Zhang Y, Guo J, Liang W, et al. Facilitating T Cell Infiltration in Tumor Microenvironment Overcomes Resistance to PD-L1 Blockade. Cancer Cell (2016) 29:285-96. doi: 10.1016/j.ccell.2016.02.004

41. Topalian SL, Taube JM, Anders RA, Pardoll DM. Mechanism-Driven Biomarkers to Guide Immune Checkpoint Blockade in Cancer Therapy. Nat Rev Cancer (2016) 16:275-87. doi: 10.1038/nrc.2016.36

42. Boutros C, Tarhini A, Routier E, Lambotte O, Ladurie FL, Carbonnel F, et al. Safety Profiles of Anti-CTLA-4 and Anti-PD-1 Antibodies Alone and in Combination. Nat Rev Clin Oncol (2016) 13:473-86. doi: 10.1038/ nrclinonc. 2016.58

43. Johnson DB, Balko JM, Compton ML, Chalkias S, Gorham J, Xu Y, et al. Fulminant Myocarditis With Combination Immune Checkpoint Blockade. N Engl J Med (2016) 375:1749-55. doi: 10.1056/NEJMoa1609214

44. Topalian SL, Hodi FS, Brahmer JR, Gettinger SN, Smith DC, McDermott DF, et al. Safety, Activity, and Immune Correlates of Anti-PD-1 Antibody in Cancer. N Engl J Med (2012) 366:2443-54. doi: 10.1056/NEJMoa1200690

45. Singh A, Peppas NA. Hydrogels and Scaffolds for Immunomodulation. Adv Mater (2014) 26:6530-41. doi: 10.1002/adma.201402105

46. Wang F, Xu D, Su H, Zhang W, Sun X, Monroe MK, et al. Supramolecular Prodrug Hydrogelator as an Immune Booster for Checkpoint Blocker-Based Immunotherapy. Sci $A d v$ (2020) 6:eaaz8985. doi: 10.1126/sciadv.aaz8985

47. Ahmed A, Tait SWG. Targeting Immunogenic Cell Death in Cancer. Mol Oncol (2020) 14:2994-3006. doi: 10.1002/1878-0261.12851

48. Mathios D, Kim JE, Mangraviti A, Phallen J, Park CK, Jackson CM, et al. Anti-PD-1 Antitumor Immunity Is Enhanced by Local and Abrogated by Systemic Chemotherapy in GBM. Sci Transl Med (2016) 8:370ral80. doi: $10.1126 /$ scitranslmed.aag2942

49. Sun Y, Feng X, Wan C, Lovell JF, Jin H, Ding J. Role of NanoparticleMediated Immunogenic Cell Death in Cancer Immunotherapy. Asian J Pharm Sci (2021) 16:129-32. doi: 10.1016/j.ajps.2020.05.004 
50. Wang C, Wang J, Zhang X, Yu S, Wen D, Hu Q, et al. In Situ Formed Reactive Oxygen Species-Responsive Scaffold With Gemcitabine and Checkpoint Inhibitor for Combination Therapy. Sci Transl Med (2018) 10: eaan3682. doi: 10.1126/scitranslmed.aan3682

51. Ruan H, Hu Q, Wen D, Chen Q, Chen G, Lu Y, et al. A Dual-Bioresponsive Drug-Delivery Depot for Combination of Epigenetic Modulation and Immune Checkpoint Blockade. Adv Mater (2019) 31:e1806957. doi: 10.1002/adma.201806957

52. Li Q, Zhao Z, Qin X, Zhang M, Du Q, Li Z, et al. A Checkpoint-Regulatable Immune Niche Created by Injectable Hydrogel for Tumor Therapy. Adv Funct Mater (2021) 31:2104630. doi: 10.1002/adfm.202104630

53. Alegre ML, Frauwirth KA, Thompson CB. T-Cell Regulation by CD28 and CTLA-4. Nat Rev Immunol (2001) 1:220-8. doi: 10.1038/35105024

54. Attia P, Phan GQ, Maker AV, Robinson MR, Quezado MM, Yang JC, et al. Autoimmunity Correlates With Tumor Regression in Patients With Metastatic Melanoma Treated With Anti-Cytotoxic T-Lymphocyte Antigen-4. J Clin Oncol (2005) 23:6043-53. doi: 10.1200/JCO.2005.06.205

55. Friedman CF, Proverbs-Singh TA, Postow MA. Treatment of the ImmuneRelated Adverse Effects of Immune Checkpoint Inhibitors: A Review. JAMA Oncol (2016) 2:1346-53. doi: 10.1001/jamaoncol.2016.1051

56. Letendre P, Monga V, Milhem M, Zakharia Y. Ipilimumab: From Preclinical Development to Future Clinical Perspectives in Melanoma. Future Oncol (2017) 13:625-36. doi: 10.2217/fon-2016-0385

57. Park YJ, Kuen DS, Chung Y. Future Prospects of Immune Checkpoint Blockade in Cancer: From Response Prediction to Overcoming Resistance. Exp Mol Med (2018) 50:1-13. doi: 10.1038/s12276-018-0130-1

58. Chung CK, Fransen MF, van der Maaden K, Campos Y, Garcia-Couce J, Kralisch D, et al. Thermosensitive Hydrogels as Sustained Drug Delivery System for CTLA-4 Checkpoint Blocking Antibodies. J Control Release (2020) 323:1-11. doi: 10.1016/j.jconrel.2020.03.050

59. Harui A, McLachlan SM, Rapoport B, Zarembinski TI, Roth MD. PeriTumor Administration of Controlled Release Anti-CTLA-4 Synergizes With Systemic Anti-PD-1 to Induce Systemic Antitumor Immunity While Sparing Autoimmune Toxicity. Cancer Immunol Immunother (2020) 69:1737-49. doi: 10.1007/s00262-020-02579-8

60. Song H, Yang P, Huang P, Zhang C, Kong D, Wang W. Injectable Polypeptide Hydrogel-Based Co-Delivery of Vaccine and Immune Checkpoint Inhibitors Improves Tumor Immunotherapy. Theranostics (2019) 9:2299-314. doi: 10.7150/thno.30577

61. Cassetta L, Pollard JW. Targeting Macrophages: Therapeutic Approaches in Cancer. Nat Rev Drug Discov (2018) 17:887-904. doi: 10.1038/nrd.2018.169

62. Pathria P, Louis TL, Varner JA. Targeting Tumor-Associated Macrophages in Cancer. Trends Immunol (2019) 40:310-27. doi: 10.1016/j.it.2019.02.003

63. Xu M, Mizoguchi I, Morishima N, Chiba Y, Mizuguchi J, Yoshimoto T. Regulation of Antitumor Immune Responses by the IL-12 Family Cytokines, IL-12, IL-23, and IL-27. Clin Dev Immunol (2010) 2010:1-9. doi: 10.1155/ 2010/832454

64. Sylvestre M, Crane CA, Pun SH. Progress on Modulating Tumor-Associated Macrophages With Biomaterials. Adv Mater (2020) 32:e1902007. doi: 10.1002/adma.201902007

65. Mizutani K, Sud S, McGregor NA, Martinovski G, Rice BT, Craig MJ, et al. The Chemokine CCL2 Increases Prostate Tumor Growth and Bone Metastasis Through Macrophage and Osteoclast Recruitment. Neoplasia (2009) 11:1235-42. doi: 10.1593/neo.09988

66. Qian BZ, Li J, Zhang H, Kitamura T, Zhang J, Campion LR, et al. CCL2 Recruits Inflammatory Monocytes to Facilitate Breast-Tumour Metastasis. Nature (2011) 475:222-5. doi: 10.1038/nature10138

67. Baay M, Brouwer A, Pauwels P, Peeters M, Lardon F. Tumor Cells and Tumor-Associated Macrophages: Secreted Proteins as Potential Targets for Therapy. Clin Dev Immunol (2011) 2011:565187. doi: 10.1155/2011/565187

68. Ries CH, Cannarile MA, Hoves S, Benz J, Wartha K, Runza V, et al. Targeting Tumor-Associated Macrophages With Anti-CSF-1R Antibody Reveals a Strategy for Cancer Therapy. Cancer Cell (2014) 25:846-59. doi: 10.1016/j.ccr.2014.05.016

69. Lee C, Bae SS, Joo H, Bae H. Melittin Suppresses Tumor Progression by Regulating Tumor-Associated Macrophages in a Lewis Lung Carcinoma Mouse Model. Oncotarget (2017) 8:54951-65. doi: 10.18632/oncotarget. 18627
70. Colombo N, Peccatori F, Paganin C, Bini S, Brandely M, Mangioni C, et al. AntiTumor and Immunomodulatory Activity of Intraperitoneal IFN-Gamma in Ovarian Carcinoma Patients With Minimal Residual Tumor After Chemotherapy. Int J Cancer (1992) 51:42-6. doi: 10.1002/ijc.2910510109

71. Beatty GL, Chiorean EG, Fishman MP, Saboury B, Teitelbaum UR, Sun W, et al. CD40 Agonists Alter Tumor Stroma and Show Efficacy Against Pancreatic Carcinoma in Mice and Humans. Science (2011) 331:1612-6. doi: 10.1126/science.1198443

72. Yang $\mathrm{G}, \mathrm{Xu} \mathrm{L}$, Chao $\mathrm{Y}, \mathrm{Xu} J$, Sun $\mathrm{X}, \mathrm{Wu} \mathrm{Y}$, et al. Hollow $\mathrm{MnO} 2$ as a TumorMicroenvironment-Responsive Biodegradable Nano-Platform for Combination Therapy Favoring Antitumor Immune Responses. Nat Commun (2017) 8:902. doi: 10.1038/s41467-017-01050-0

73. Andon FT, Digifico E, Maeda A, Erreni M, Mantovani A, Alonso MJ, et al. Targeting Tumor Associated Macrophages: The New Challenge for Nanomedicine. Semin Immunol (2017) 34:103-13. doi: 10.1016/ j.smim.2017.09.004

74. Bonapace L, Coissieux MM, Wyckoff J, Mertz KD, Varga Z, Junt T, et al. Cessation of CCL2 Inhibition Accelerates Breast Cancer Metastasis by Promoting Angiogenesis. Nature (2014) 515:130-3. doi: 10.1038/nature13862

75. Hume DA, MacDonald KP. Therapeutic Applications of Macrophage Colony-Stimulating Factor-1 (CSF-1) and Antagonists of CSF-1 Receptor (CSF-1R) Signaling. Blood (2012) 119:1810-20. doi: 10.1182/blood-2011-09379214

76. Jin H, Wan C, Zou Z, Zhao G, Zhang L, Geng Y, et al. Tumor Ablation and Therapeutic Immunity Induction by an Injectable Peptide Hydrogel. ACS Nano (2018) 12:3295-310. doi: 10.1021/acsnano.7b08148

77. Rodell CB, Ahmed MS, Garris CS, Pittet MJ, Weissleder R. Development of Adamantane-Conjugated TLR7/8 Agonists for Supramolecular Delivery and Cancer Immunotherapy. Theranostics (2019) 9:8426-36. doi: 10.7150/ thno. 35434

78. Dai X, Meng J, Deng S, Zhang L, Wan C, Lu L, et al. Targeting CAMKII to Reprogram Tumor-Associated Macrophages and Inhibit Tumor Cells for Cancer Immunotherapy With an Injectable Hybrid Peptide Hydrogel. Theranostics (2020) 10:3049-63. doi: 10.7150/thno.42385

79. Chen P, Zuo H, Xiong H, Kolar MJ, Chu Q, Saghatelian A, et al. Gpr132 Sensing of Lactate Mediates Tumor-Macrophage Interplay to Promote Breast Cancer Metastasis. Proc Natl Acad Sci USA (2017) 114:580-5. doi: 10.1073/pnas.1614035114

80. Yang M, McKay D, Pollard JW, Lewis CE. Diverse Functions of Macrophages in Different Tumor Microenvironments. Cancer Res (2018) 78:5492-503. doi: 10.1158/0008-5472.CAN-18-1367

81. Liao ZX, Fa YC, Kempson IM, Tseng SJ. Repolarization of M2 to M1 Macrophages Triggered by Lactate Oxidase Released From Methylcellulose Hydrogel. Bioconjug Chem (2019) 30:2697-702. doi: 10.1021/ acs.bioconjchem.9b00618

82. Muraoka D, Seo N, Hayashi T, Tahara Y, Fujii K, Tawara I, et al. Antigen Delivery Targeted to Tumor-Associated Macrophages Overcomes Tumor Immune Resistance. J Clin Invest (2019) 129:1278-94. doi: 10.1172/JCI97642

83. Yeung OW, Lo CM, Ling CC, Qi X, Geng W, Li CX, et al. Alternatively Activated (M2) Macrophages Promote Tumour Growth and Invasiveness in Hepatocellular Carcinoma. J Hepatol (2015) 62:607-16. doi: 10.1016/ j.jhep.2014.10.029

84. Guerra AD, Yeung OWH, Qi X, Kao WJ, Man K. The Anti-Tumor Effects of M1 Macrophage-Loaded Poly (Ethylene Glycol) and Gelatin-Based Hydrogels on Hepatocellular Carcinoma. Theranostics (2017) 7:3732-44. doi: $10.7150 /$ thno.20251

85. Martin JD, Seano G, Jain RK. Normalizing Function of Tumor Vessels: Progress, Opportunities, and Challenges. Annu Rev Physiol (2019) 81:50534. doi: 10.1146/annurev-physiol-020518-114700

86. Viallard C, Larrivee B. Tumor Angiogenesis and Vascular Normalization: Alternative Therapeutic Targets. Angiogenesis (2017) 20:409-26. doi: 10.1007/s10456-017-9562-9

87. Goel S, Duda DG, Xu L, Munn LL, Boucher Y, Fukumura D, et al. Normalization of the Vasculature for Treatment of Cancer and Other Diseases. Physiol Rev (2011) 91:1071-121. doi: 10.1152/physrev.00038.2010

88. Jain RK. Antiangiogenesis Strategies Revisited: From Starving Tumors to Alleviating Hypoxia. Cancer Cell (2014) 26:605-22. doi: 10.1016/ j.ccell.2014.10.006 
89. Ferrara N, Gerber HP, LeCouter J. The Biology of VEGF and Its Receptors. Nat Med (2003) 9:669-76. doi: 10.1038/nm0603-669

90. Huang Y, Yuan J, Righi E, Kamoun WS, Ancukiewicz M, Nezivar J, et al. Vascular Normalizing Doses of Antiangiogenic Treatment Reprogram the Immunosuppressive Tumor Microenvironment and Enhance Immunotherapy. Proc Natl Acad Sci USA (2012) 109:17561-6. doi: 10.1073/pnas.1215397109

91. Shrimali RK, Yu Z, Theoret MR, Chinnasamy D, Restifo NP, Rosenberg SA. Antiangiogenic Agents can Increase Lymphocyte Infiltration Into Tumor and Enhance the Effectiveness of Adoptive Immunotherapy of Cancer. Cancer Res (2010) 70:6171-80. doi: 10.1158/0008-5472.CAN-10-0153

92. Ferreira NN, MBF L, Miranda-Goncalves V, Reis RM, Seraphim TV, Borges JC, et al. Alginate Hydrogel Improves Anti-Angiogenic Bevacizumab Activity in Cancer Therapy. Eur J Pharm Biopharm (2017) 119:271-82. doi: 10.1016/j.ejpb.2017.06.028

93. Zhou TJ, Xing L, Fan YT, Cui PF, Jiang HL. Inhibition of Breast Cancer Proliferation and Metastasis by Strengthening Host Immunity With a Prolonged Oxygen-Generating Phototherapy Hydrogel. J Control Release (2019) 309:82-93. doi: 10.1016/j.jconrel.2019.07.028

94. Gartel AL, Kandel ES. RNA Interference in Cancer. Biomol Eng (2006) 23:17-34. doi: 10.1016/j.bioeng.2006.01.002

95. Miele E, Spinelli GP, Miele E, Di Fabrizio E, Ferretti E, Tomao S, et al. Nanoparticle-Based Delivery of Small Interfering RNA: Challenges for Cancer Therapy. Int J Nanomed (2012) 7:3637-57. doi: 10.2147/IJN.S23696

96. Zamore PD, Tuschl T, Sharp PA, Bartel DP. RNAi: Double-Stranded RNA Directs the ATP-Dependent Cleavage of mRNA at 21 to 23 Nucleotide Intervals. Cell (2000) 101:25-33. doi: 10.1016/S0092-8674(00)80620-0

97. Fujii H, Shin-Ya M, Takeda S, Hashimoto Y, Mukai SA, Sawada S, et al. Cycloamylose-Nanogel Drug Delivery System-Mediated Intratumor Silencing of the Vascular Endothelial Growth Factor Regulates Neovascularization in Tumor Microenvironment. Cancer Sci (2014) 105:1616-25. doi: 10.1111/cas.12547

98. Ebos JM, Kerbel RS. Antiangiogenic Therapy: Impact on Invasion, Disease Progression, and Metastasis. Nat Rev Clin Oncol (2011) 8:210-21. doi: 10.1038/nrclinonc.2011.21

99. Huang Y, Goel S, Duda DG, Fukumura D, Jain RK. Vascular Normalization as an Emerging Strategy to Enhance Cancer Immunotherapy. Cancer Res (2013) 73:2943-8. doi: 10.1158/0008-5472.CAN-12-4354

100. Pal S, Medatwal N, Kumar S, Kar A, Komalla V, Yavvari PS, et al. A Localized Chimeric Hydrogel Therapy Combats Tumor Progression Through Alteration of Sphingolipid Metabolism. ACS Cent Sci (2019) 5:1648-62. doi: 10.1021/acscentsci.9b00551

101. Yu S, Wei S, Liu L, Qi D, Wang J, Chen G, et al. Enhanced Local Cancer Therapy Using a CA4P and CDDP Co-Loaded Polypeptide Gel Depot. Biomater Sci (2019) 7:860-6. doi: 10.1039/c8bm01442f

102. Chen J, Luo H, Liu Y, Zhang W, Li H, Luo T, et al. Oxygen-Self-Produced Nanoplatform for Relieving Hypoxia and Breaking Resistance to Sonodynamic Treatment of Pancreatic Cancer. ACS Nano (2017) 11:12849-62. doi: 10.1021/acsnano.7b08225

103. Li SY, Cheng H, Xie BR, Qiu WX, Zeng JY, Li CX, et al. Cancer Cell Membrane Camouflaged Cascade Bioreactor for Cancer Targeted Starvation and Photodynamic Therapy. ACS Nano (2017) 11:7006-18. doi: 10.1021/ acsnano.7b02533

104. Zhang K, Fang Y, He Y, Yin H, Guan X, Pu Y, et al. Extravascular Gelation Shrinkage-Derived Internal Stress Enables Tumor Starvation Therapy With Suppressed Metastasis and Recurrence. Nat Commun (2019) 10:5380. doi: 10.1038/s41467-019-13115-3

105. Marabelle A, Kohrt H, Sagiv-Barfi I, Ajami B, Axtell RC, Zhou G, et al. Depleting Tumor-Specific Tregs at a Single Site Eradicates Disseminated Tumors. J Clin Invest (2013) 123:2447-63. doi: 10.1172/JCI64859

106. Deng C, Zhang Q, Jia M, Zhao J, Sun X, Gong T, et al. Tumors and Their Microenvironment Dual-Targeting Chemotherapy With Local Immune Adjuvant Therapy for Effective Antitumor Immunity Against Breast Cancer. Adv Sci (Weinh) (2019) 6:1801868. doi: 10.1002/advs.201801868

107. Dong X, Yang A, Bai Y, Kong D, Lv F. Dual Fluorescence Imaging-Guided Programmed Delivery of Doxorubicin and CpG Nanoparticles to Modulate Tumor Microenvironment for Effective Chemo-Immunotherapy. Biomaterials (2020) 230:119659. doi: 10.1016/j.biomaterials.2019.119659
108. Ghiringhelli F, Larmonier N, Schmitt E, Parcellier A, Cathelin D, Garrido C, et al. CD4+CD25+ Regulatory T Cells Suppress Tumor Immunity But Are Sensitive to Cyclophosphamide Which Allows Immunotherapy of Established Tumors to be Curative. Eur J Immunol (2004) 34:336-44. doi: 10.1002/eji.200324181

109. Ghiringhelli F, Menard C, Puig PE, Ladoire S, Roux S, Martin F, et al. Metronomic Cyclophosphamide Regimen Selectively Depletes CD4+CD25+ Regulatory T Cells and Restores T and NK Effector Functions in End Stage Cancer Patients. Cancer Immunol Immunother (2007) 56:641-8. doi: 10.1007/s00262-006-0225-8

110. Lutsiak ME, Semnani RT, De Pascalis R, Kashmiri SV, Schlom J, Sabzevari H. Inhibition of $\mathrm{CD} 4(+) 25+\mathrm{T}$ Regulatory Cell Function Implicated in Enhanced Immune Response by Low-Dose Cyclophosphamide. Blood (2005) 105:2862-8. doi: 10.1182/blood-2004-06-2410

111. Rettig L, Seidenberg S, Parvanova I, Samaras P, Curioni A, Knuth A, et al. Gemcitabine Depletes Regulatory T-Cells in Human and Mice and Enhances Triggering of Vaccine-Specific Cytotoxic T-Cells. Int J Cancer (2011) 129:832-8. doi: 10.1002/ijc.25756

112. Vincent J, Mignot G, Chalmin F, Ladoire S, Bruchard M, Chevriaux A, et al. 5-Fluorouracil Selectively Kills Tumor-Associated Myeloid-Derived Suppressor Cells Resulting in Enhanced T Cell-Dependent Antitumor Immunity. Cancer Res (2010) 70:3052-61. doi: 10.1158/0008-5472.CAN09-3690

113. Seo SH, Han HD, Noh KH, Kim TW, Son SW. Chitosan Hydrogel Containing GMCSF and a Cancer Drug Exerts Synergistic Anti-Tumor Effects via the Induction of CD8+ T Cell-Mediated Anti-Tumor Immunity. Clin Exp Metastasis (2009) 26:179-87. doi: 10.1007/s10585-008-9228-5

114. Bos GW, Jacobs JJ, Koten JW, Van Tomme S, Veldhuis T, van Nostrum CF, et al. In Situ Crosslinked Biodegradable Hydrogels Loaded With IL-2 Are Effective Tools for Local IL-2 Therapy. Eur J Pharm Sci (2004) 21:561-7. doi: 10.1016/j.ejps.2003.12.007

115. Xu K, Lee F, Gao SJ, Chung JE, Yano H, Kurisawa M. Injectable Hyaluronic Acid-Tyramine Hydrogels Incorporating Interferon-Alpha2a for Liver Cancer Therapy. J Control Release (2013) 166:203-10. doi: 10.1016/ j.jconrel.2013.01.008

116. Oh E, Oh JE, Hong J, Chung Y, Lee Y, Park KD, et al. Optimized Biodegradable Polymeric Reservoir-Mediated Local and Sustained CoDelivery of Dendritic Cells and Oncolytic Adenovirus Co-Expressing IL12 and GM-CSF for Cancer Immunotherapy. J Control Release (2017) 259:115-27. doi: 10.1016/j.jconrel.2017.03.028

117. Coussens LM, Zitvogel L, Palucka AK. Neutralizing Tumor-Promoting Chronic Inflammation: A Magic Bullet? Science (2013) 339:286-91. doi: $10.1126 /$ science. 1232227

118. Gendy AS, Lipskar A, Glick RD, Steinberg BM, Edelman M, Soffer SZ. Selective Inhibition of Cyclooxygenase-2 Suppresses Metastatic Disease Without Affecting Primary Tumor Growth in a Murine Model of Ewing Sarcoma. J Pediatr Surg (2011) 46:108-14. doi: 10.1016/j.jpedsurg.2010.09.074

119. Kawamori T, Rao CV, Seibert K, Reddy BS. Chemopreventive Activity of Celecoxib, A Specific Cyclooxygenase-2 Inhibitor, Against Colon Carcinogenesis. Cancer Res (1998) 58:409-12.

120. Li Y, Fang M, Zhang J, Wang J, Song Y, Shi J, et al. Hydrogel Dual Delivered Celecoxib and Anti-PD-1 Synergistically Improve Antitumor Immunity. Oncoimmunology (2016) 5:e1074374. doi: 10.1080/2162402X.2015.1074374

121. Wang N, Gao Q, Tang J, Jiang Y, Yang L, Shi X, et al. Anti-Tumor Effect of Local Injectable Hydrogel-Loaded Endostatin Alone and in Combination With Radiotherapy for Lung Cancer. Drug Deliv (2021) 28:183-94. doi: 10.1080/10717544.2020.1869864

122. Tan B, Huang L, Wu Y, Liao J. Advances and Trends of Hydrogel Therapy Platform in Localized Tumor Treatment: A Review. J BioMed Mater Res A (2021) 109:404-25. doi: 10.1002/jbm.a.37062

123. Wang $\mathrm{M}$, Chen $\mathrm{M}$, Niu W, Winston DD, Cheng W, Lei B. Injectable Biodegradation-Visual Self-Healing Citrate Hydrogel With High Tissue Penetration for Microenvironment-Responsive Degradation and Local Tumor Therapy. Biomaterials (2020) 261:120301. doi: 10.1016/j. biomaterials.2020.120301

124. Wan C, Sun Y, Hu Y, Huang J, Lu L, Gao Y, et al. Peptide Hydrogels Loaded With Irradiated Tumor Cell Secretions Enhance Cancer Immunotherapy. Nano Today (2021) 41:101323. doi: 10.1016/j.nantod.2021.101323 
125. Ma H, He C, Chen X. Injectable Hydrogels as Local Depots at Tumor Sites for Antitumor Immunotherapy and Immune-Based Combination Therapy. Macromol Biosci (2021) 21:e2100039. doi: 10.1002/mabi. 202100039

126. Sun Y, Hu Y, Wan C, Lovell JF, Jin H, Yang K. Local Biomaterial-Assisted Antitumour Immunotherapy for Effusions in the Pleural and Peritoneal Cavities Caused by Malignancies. Biomater Sci (2021) 9:6381-90. doi: $10.1039 / \mathrm{d} 1 \mathrm{bm} 00971 \mathrm{k}$

Conflict of Interest: The authors declare that the research was conducted in the absence of any commercial or financial relationships that could be construed as a potential conflict of interest.
Publisher's Note: All claims expressed in this article are solely those of the authors and do not necessarily represent those of their affiliated organizations, or those of the publisher, the editors and the reviewers. Any product that may be evaluated in this article, or claim that may be made by its manufacturer, is not guaranteed or endorsed by the publisher.

Copyright (C) 2022 Liu, Geng, Yue, Lo, Huang and Jin. This is an open-access article distributed under the terms of the Creative Commons Attribution License (CC BY). The use, distribution or reproduction in other forums is permitted, provided the original author(s) and the copyright owner(s) are credited and that the original publication in this journal is cited, in accordance with accepted academic practice. No use, distribution or reproduction is permitted which does not comply with these terms. 